\title{
Meningitis simultánea por candida y tuberculosis como debut de sida
}

\author{
M. ARIAS GÓMEZ, I. REQUENA CABALLERO, C. LEMA DEVESA, \\ J. SUÁREZ DONO*, J. LLOVO MARTÍNEZ**, V. MARTINO** \\ Servicios de Neurología, *Medicina Interna y **Microbiología. Hospital de Conxo. \\ Departamento de Medicina. Complexo Hospitalario Universitario. \\ Santiago de Compostela.
}

SIMULTANEOUS MENINGITIS CAUSED BY CANDIDA AND TUBER CULOSIS AS AIDS MANIFESTATION

\begin{abstract}
RESUMEN
Las meningoencefalitis por gérmenes oportunistas ocupan un lugar importante dentro de la patología neurológica del sida. Treponema palli dum y Mycobacterium tuberculosis dentro de las baceterias, Crytptococ cus neoformans dentro de los hongos, Toxoplasma gondii dentro de los protozoos y el Papovavirus $J C$ dentro de los virus, son los gérmenes más frecuentes dentro de cada grupo. Con cierta frecuencia las infecciones son mixtas, tal como ocurrió en el caso que se presenta de meningitis simultánea por Candida albicans y Mycobacterium tuberculosis, que cursó con pleocitosis neutrófila y normoglucorraquia, constituyendo la primera manifestación de un sida. El estudio secuencial del LCR es clave para el diagnóstico de estos casos, que, pese a su gravedad, pueden tener un tratamiento efectivo, tal como ocurrió en el que se describe. Es el primer caso descrito de meningitis simultánea candidiásica y tuberculosa en paciente portador de VIH.
\end{abstract}

PALABRAS CLAVE: Sida. Meningitis. Candida. Tuberculosis.

\begin{abstract}
Opportunistic germs meningoencephalitis plays an important role within neurologic pathology in aids. Treponema pallidum and Mycobac terium tuberculosis among bacteries, Cryptococcus neoformans in fungus group, Toxoplasma gondii in protozoos group and Papovavirus $J C$ in virus one are the most frequently implicated germs. Sometimes infections are mixed. We present a simultaneous meningitis case produced by Candida albicans and Mycobacterium tuberculosis that coursed with neutrophilic pleocytosis in CSF and normal glucose CSF levels, consisting the clinical debut of aids. Repeated CSF examinations are the diagnostic clue owing, as in our case, instauration of early treatment. Present case of simultaneous tuberculous and candidiasic meningitis is the first one described in a HIV positive patient.
\end{abstract}

KEY WORDS: Aids. Meningitis. Candida. Tuberculosis.

Arias Gómez M, Requena Caballero I, Lema Devesa C, Suárez Dono J, Llovo Martínez J, Martino V. Meningitis simultánea por Candida y tuberculosis como debut de sida. An Med Interna (Madrid) 2001; 18: 479-480.

\section{INTRODUCCIÓN}

Dentro del amplio espectro de afectación neurológica que pueden presentar los pacientes infectados por VIH, las infecciones por gérmenes oportunistas constituyen un capítulo importante: Treponema pallidum y Mycobacterium tuberculo sis (bacterias), Cryptococcus neoformans (hongos), Papovavi rus $J C$, productor de leucoencefalopatía multifocal progresiva, (virus) y Toxoplasma gondii (protozoos) son, hablando en términos de frecuencia, los jefes de fila dentro de cada grupo $(1,2)$. Aunque no son excepcionales las infecciones mixtas, no hemos encontrado ningún caso en la literatura de meningitis simultánea por Candida albicans y Mycobacterium tuber culosis en pacientes con infección por el VIH, tal como ocurrió en el paciente que se presenta, constituyendo además la forma de presentación de un sida.

\section{CASO APORTADO}

Varón de 42 años de edad, fontanero de profesión y soltero, que ingresó por cuadro de cefalea, cambio de carácter y trastorno del comportamiento, de poco más de una semana de evolución. En los dos últimos meses, venía presentando astenia, anorexia y sudoración nocturna; había adelgazado unos $12 \mathrm{~kg}$. Entre sus antecedentes señalar traumatismo craneofacial, que tuvo lugar 15 años antes, con importante hemorragia, que requirió transfusión sanguínea. Reconocía contactos heterosexuales episódicos. En el último año recibió tratamiento esteroideo tópico por eczema seborreico facial.

En la exploración clínica general inicial destacaba temperatura de $37,5^{\circ} \mathrm{C}$, lesiones orofaríngeas sugestivas de cándida, eritema descamativo facial, desnutrición importante y crepitantes bilaterales en la auscultación pulmonar. No se palpaban adenopatías valorables. En el examen neurológico se comprobó desorientación temporoespacial, deterioro de memoria reciente y anterógrada con fabulación, disartria y rigidez de nuca con signos de Kernig y Brudzinski.

Trabajo aceptado: 28 de diciembre de 1999

Correspondencia: M. Arias Gómez. C/ Ramón Piñeiro 5-2 ${ }^{\circ}$ C. 15706. Santiago de Compostela. 
Con carácter urgente, se le practicaron los siguientes estudios: analítica de sangre, que reveló moderada anemia microcítica e hiponatremia; radiología de tórax, que puso de manifiesto patrón intersticial difuso; TAC cerebral, que demostró leve dilatación del sistema ventricular supratentorial; estudio de LCR, en el que se encontraron 1500 células (neutrófilos) por $\mathrm{mm}^{3}, 1,7 \mathrm{~g} / \mathrm{l}$ de proteínas y $126 \mathrm{mg} / \mathrm{dl}$ de glucosa (156 mg/dl en sangre), observándose esporas germinadas de Can dida sp. En este primer estudio no se realizó tinción para BAAR. Fue ingresado y puesto a tratamiento con amfotericina B y cefotaxima.

Durante el ingreso se realizaron diversos estudios analíticos de sangre, destacando serología de VIH1 positiva, siendo la carga viral determinada por PCR de > de 100.000 copias/ml de ARN-VIH1; CD4 de 185 por $\mathrm{mm}^{3}$. Se repitió el estudio de LCR, resultando positiva la tinción para BAAR. Se suprimió la cefotaxima y se añadieron al tratamiento isoniazida, pirazinamida, etambutol y rifampicina. En los cultivos del LCR creció Candida albicans y también, a las 4 semanas, Mycobacterium tuberculosis en el Löwenstein.

El paciente presentó, como complicaciones, insuficiencia respiratoria y síndrome de secreción inadecuada de $\mathrm{ADH}$, pero finalmente su evolución fue satisfactoria y pudo ser dado de alta sin secuelas a las 6 semanas del ingreso, prosiguiendo con fluconazol por vía oral y los tuberculostáticos.

\section{DISCUSIÓN}

Nuestro paciente presentó un cuadro de meningitis mixta, por cándida y tuberculosis, de evolución subaguda, en el que habría que subrayar de entrada dos aspectos: a) la pleocitosis neutrófila inicial con normoglucorraquia; b) la observación directa de las esporas fúngicas en el LCR. El cuestionarnos el diagnóstico inicial, de meningitis candidiásica y bacteriana, motivó la realización del segundo estudio del LCR, que sirvió para demostrar la presencia de bacilo tuberculoso y poder instaurar un tratamiento efectivo.

La meningitis por candida, en términos generales, es una entidad rara, que se presenta con mayor frecuencia en pacientes neuroquirúrgicos, algunos de ellos portadores de válvulas de derivación $(3,4)$; también puede seguir a cuadros de meningitis bacteriana, tratados con antibioterapia de amplio espectro (5); existen descritas formas crónicas y casos con pleocitosis neutrófila (6).

\section{Bibliografía}

1. Spencer PM, Jackson GG. Fungal and mycobacterial infections in patients infected with the human immunodeficiency virus. J Antimicrob Chemother 1989; 23 (Suppl A): 107-125.

2. Berger JR, McArthur JC. The neurological manifestations of human immunodeficiency virus infection. En: Bradley WG, Daroff RB, Fenichel GM, Marsden D. (edits), Neurology in Clinical Practice. Boston. Butterwoeth-Heinemann 1996: 1276-1292.

3. Nguyen MH, Yu VL. Meningitis caused by Candida species: an emerging problem in neurosurgical patients. Clin Infect Dis 1995; 21: 323-327.

4. Sánchez-Portocarrero J, Martín-Rabadán P, Saldaña CJ, Pérez-Cecilia E. Candida cerebrospinal fluid shunt infection. Report of two new cases and review of the literature. Diagn Microbiol Infect Dis 1994; 20: 33-40.

5. Gelfand MS, McGee ZA, Kaise AB, Tally FP, Moses J. Candidal meningitis following bacterial meningitis. South Med J 1990; 83: 567-570.

6. Voice RA, Bradley SF, Sangeorzan JA, Kauffman CA. Chronic candidal meningitis: an uncommon manifestation of meningitis. Clin Infect Dis 1994; 19: 60-66.

7. Cavaliere MJ, Maeda MY, Longatto Filho A, Shirata NK, Santos RT, Kitamura C, Ueki SY, Martins MC. Frequency of Candida sp infection in tuberculous patients with acquired immunodeficiency syndrome:
Los pacientes con sida llegan a tener algún tipo de infección por cándida en más del $80 \%$ de los casos, pero la meningitis candidiásica es mucho menos frecuente que la meningitis por criptococo (7). Casado y cols. en 1997 (8) revisan la literatura, encontrando 14 casos (5 propios) de meningitis por cándida, señalando que la cefalea y fiebre sin signos neurológicos focales constituyen el cuadro clínico habitual; el LCR suele ser similar al de la meningitis tuberculosa; tiene una mortalidad situada alrededor del 31\%; los CD4 suelen estar por debajo de 140 por $\mathrm{mm}^{3}$; la amfotericina B es el tratamiento de elección, debiendo asociarse fluocitosina en caso de mala evolución y reservando el fluconazol para mantenimiento.

En la meningitis tuberculosa del paciente con sida, el curso clínico, las características del LCR y la mortalidad intrahospitalaria son similares a la meningitis tuberculosa de los pacientes VIH negativos. No obstante, como forma particular de tuberculosis, la meningitis es cinco veces más frecuente en los tuberculosos VIH positivos que en los que no lo son (9). En algunas series la meningitis tuberculosa, de los pacientes VIH positivos, se asocia a una mayor probabilidad de hallazgo de masas intracraneales (10). La meningitis tuberculosa es el primer tipo de meningitis que se asocia con la infección del VIH adquirida mediante inyección endovenosa de drogas (11). Al igual que la meningitis por candida, la meningitis tuberculosa puede presentarse con pleocitosis de predominio polimorfonuclear, lo que supone un signo de gravedad .

Pese a que las infecciones mixtas son relativamente comunes en el sida y pese a que as infecciones oportunistas extraneurológicas por candida y micobacterias son las más frecuentes en este tipo de pacientes $(1,7)$, no hemos encontrado ningún caso de meningitis simultánea por candida y tuberculosis en pacientes infectados por VIH y sólo un caso infantil descrito en 1981 (12).

La conclusión no puede ser otra que, ante pacientes inmunodeprimidos con meningitis atípicas o de mala evolución, el estudio secuencial del LCR, investigando la posibilidad de meningitis por varios gérmenes, debe ser mandatorio; sería recomendable utilizar técnicas de biología molecular si las tinciones resultan negativas, ya que, en el caso particular de la tuberculosis, el esperar al Löwenstein no resultaría operativo. morphological and immunocytochemical study in sputum. Pathologica 1994; 86: 409-411.

8. Casado JL, Quereda C, Oliva J, Navas E, Moreno A, Pintado V, Cobo J, Corral I. Candidal meningitis in HIV-infected patients: analysis of 14 cases. Clin Infect Dis 1997; 25: 673-676.

9. Berenguer J, Moreno S, Laguna F, Vicente T, Adrados M, Ortega A, González-LaHoz J, Bouza F. Tuberculous meningitis in patients infected with the human immunodeficiency virus. N Engl J Med 1992; 326: 668-672.

10. Dube MP, Holtom PD, Larsen RA. Tuberculous meningitis in patients with and without human immunodeficiency virus infection. Am J Med 1992; 93: 520-524.

11. Sanchez-Portocarrero J, Pérez-Cecilia E, Jiménez-Escrig A, MartinRabadan P, Roca V, Ruíz Yagüe M, Romero-Vivas J, Palau E, Picazo JJ. Tuberculous meningitis. Clinical characteristics and comparison with criptococcal meningitis in patients with human immunodeficiency virus infection. Arch Neurol 1996; 53: 671-676.

12. Popow C, Haschke F, Maida-E, Kristoferitsch W, Schilling R, Gotz M, Groh C. Kombinierte Tbc und Candidameningitis bei einem achtjahrigen Knaben. Klin Padiatr 1981; 193: 401-3. 